Folia Cardiologica 2021 vol. 16, no. 3, pages 191-197 DOI: $10.5603 / F C .2021 .0022$ Copyright (C) 2021 Via Medica ISSN 2353-7752 e-ISSN 2353-7760

\title{
Clinician's guide for dapagliflozin use in heart failure with reduced ejection fraction
}

\author{
Małgorzata Lelonek (iD \\ Department of Noninvasive Cardiology, Medical University of Lodz, Łódź, Poland
}

\section{Abstract}

Dapagliflozin belongs to a new class of drugs used for the treatment of heart failure - sodium-glucose cotransporter type 2 inhibitors (SGLT2i). Based on the DAPA-HF study results, dapagliflozin has become the first SGLT2i approved for the treatment of symptomatic chronic heart failure with reduced ejection fraction. The present review summarizes the most important clinical issues related to the treatment with this drug.

Key words: heart failure with reduced ejection fraction, SGLT2 inhibitors, dapagliflozin

Folia Cardiologica 2021; 16, 3: 191-197

\section{Introduction}

Heart failure (HF) is present in about 1.2 million patients in Poland [1]. The population of patients with HF continues to grow and poses an increasing financial burden, mostly related to hospitalizations due to $\mathrm{HF}$ exacerbation. Of note, effective treatment for HF and preventing its progression are possible with innovative drugs that, shortly after their introduction, have been shown to bring significant clinical benefits including a reduction in the risk of cardiovascular mortality and admissions due to HF. One of these therapies is dapagliflozin.

\section{The mechanism of action}

\section{of sodium-glucose cotransporter type 2} inhibitors (SGLT2i)

Inhibitors of the sodium-glucose cotransporter type 2, which is present in the proximal renal tubule, results in urinary glucose excretion by reducing glucose reabsorption and lowering the renal glucose threshold [2]. It is the basic mechanism of action of these drugs in the treatment of diabetes. Glucosuria results in osmotic diuresis and is associated with a negative energy balance, which leads to weight reduction and improves insulin sensitivity [3]. This effect is insulin-independent and is not associated with a risk of hypoglycaemia.

Other beneficial mechanism of action of SGLT2i which are particularly important in HF include a reduction of sodium reabsorption in the renal tubule [4]. The resultant increased natriuresis and osmotic diuresis, plasma volume reduction and blood pressure lowering lead to a reduction in left ventricular preload and afterload. At the same time, an increased sodium load reaching the macula densa decreases activation of the sympathetic system and the renin-angiotensin-aldosterone (RAA) system. In addition, increased production and use of ketone bodies in the myocardium improve myocardial metabolism and inhibit myocardial remodelling [4]. Another beneficial effect of SGLT2i is a nephroprotective effect resulting from afferent arteriolar constriction, leading to a reduction of glomerular hyperfiltration and urinary albumin excretion [5].

Address for correspondence: Małgorzata Lelonek Professor, MD, PhD, FESC, FHF, Zakład Kardiologii Nieinwazyjnej, Katedra Chorób Wewnętrznych i Kardiologii, Uniwersytet Medyczny w Łodzi, ul. Żeromskiego 113, 90-549 Łódź, Poland, e-mail: malgorzata.lelonek@umed.lodz.pl

This article is available in open access under Creative Common Attribution-Non-Commercial-No Derivatives 4.0 International (CC BY-NC-ND 4.0) license, allowing to download articles and share them with others as long as they credit the authors and the publisher, but without permission to change them in any way or use them commercially. 
Table 1. Inclusion and exclusion criteria in the DAPA-HF study (source [6])

\section{Major inclusion criteria}

Men and women $\geq 18$ years of age, with diabetes type 2 or without diabetes

Documented diagnosis of symptomatic HFrEF for $\geq 2$ months (NYHA class II-IV)

LVEF $\leq 40 \%$ within the last 12 months

Increased NT-proBNP level ( $\geq 600 \mathrm{pg} / \mathrm{mL}$, or $\geq 400 \mathrm{pg} / \mathrm{mL}$ if hospitalization for HF within 12 months, or $\geq 900 \mathrm{pg} / \mathrm{mL}$ if atrial fibrillation/flutter was diagnosed, regardless of the history of hospitalization for HF)

Optimal standard drug treatment for $\mathrm{HF}$ and device therapy (cardioverter-defibrillator and/or cardiac resynchronization therapy)

Optimal and stable (for $\geq 4$ months) standard drug treatment for HFrEF according to the local guidelines (unless contraindicated or not tolerated), including ACEI/ARB or ARNI, beta-blocker, and MRA if indicated

eGFR $\geq 30 \mathrm{~mL} / \mathrm{min} / 1.73 \mathrm{~m}^{2}$

Major exclusion criteria

Treatment with SGLT2i within 8 weeks prior to study inclusion or SGLT2i intolerance

Diabetes type 1

Symptomatic hypotension or systolic blood pressure $<95 \mathrm{~mm} \mathrm{Hg}$

Current acute exacerbated HF or hospitalization due to exacerbated HF within the last 4 weeks

Myocardial revascularization (PCl or CABG), valve repair/replacement, implantation of a cardiac pacemaker (CRT) within the last 12 weeks or such procedure planned in the post-randomization period

HF due to restrictive cardiomyopathy, active myocarditis, constrictive pericarditis, hypertrophic cardiomyopathy, or uncorrected primary valve disease

eGFR $<30 \mathrm{~mL} / \mathrm{min} / 1.73 \mathrm{~m}^{2}$ or rapidly worsening renal function

HFrEF - heart failure with reduced ejection fraction; NYHA - New York Heart Association; LVEF - left ventricular ejection fraction; NT-proBNP - N-terminal pro-B-type natriuretic peptide; HF - heart failure; ACEI - angiotensin-converting enzyme inhibitor; ARB - angiotensin receptor blocker; ARNI - angiotensin receptor blocker and neprilysin inhibitor; MRA - mineralocorticoid receptor antagonist; eGFR - estimated glomerular filtration rate; SGLT2i - sodium-glucose cotransporter type 2 inhibitor; PCI - percutaneous coronary intervention; CABG -, coronary artery bypass grafting; CRT - cardiac resynchronization therapy

\section{DAPA-HF study results}

The clinical efficacy of dapagliflozin in the treatment of symptomatic chronic heart failure with reduced ejection fraction (HFrEF) has been documented in the Dapagliflozin and Prevention of Adverse Outcomes in Heart Failure (DAPA-HF) study, a prospective multicentre randomized placebo-controlled phase III trial to evaluate the efficacy and safety of dapagliflozin compared to placebo [6]. Dapagliflozin was added to standard guideline-recommended HFrEF therapy, i.e., an angiotensin-converting enzyme inhibitor (ACEI), angiotensin receptor blocker (ARB) or an angiotensin receptor blocker and neprilysin inhibitor (ARNI), beta-blocker, and/or mineralocorticoid receptor antagonist (MRA) in stable doses for at least 4 weeks. The study included 4,744 symptomatic New York Heart Association (NYHA) class II-IV patients with chronic HF and left ventricular ejection fraction $\leq 40 \%$. Both patients with diabetes type 2 and non-diabetic ones were recruited to the study and randomized in the 1:1 ratio to dapagliflozin $10 \mathrm{mg}$ once daily or placebo. The DAPA-HF study was the first dapagliflozin trial that included non-diabetic patients and those with renal dysfunction, i.e., with the estimated glomerular filtration rate (eGFR) of $30-60 \mathrm{~mL} /$ $/ \mathrm{min} / 1.73 \mathrm{~m}^{2}$.
The inclusion and exclusion criteria are shown in Table 1. Of note, the patients included in the DAPA-HF study received optimal $\mathrm{HF}$ treatment, as $94 \%$ were treated with ACEI/ARB/ARNI (including 11\% treated with ARNI), 96\% with a beta-blocker, and $71 \%$ with a MRA. Compared to earlier studies in HFrEF, more patients in the DAPA-HF study were treated with an implanted cardiac device, including an implantable cardioverter-defibrillator (ICD) in 26\%, and cardiac resynchronization therapy (CRT) in $8 \%$.

Compared to placebo, dapagliflozin treatment was associated with a $26 \%$ lower risk of the primary endpoint of cardiovascular death, hospitalization for $\mathrm{HF}$, or an urgent HF-related visit without hospitalization $(p<0.0001) d u-$ ring the median follow-up of 18.2 months (Table 2). The reduction in the primary endpoint rate was documented as early as at $\mathbf{2 8}$ days of treatment [hazard ratio (HR) $0.51,95 \%$ confidence interval $(\mathrm{Cl}): 0.28-0.94, \mathrm{p}=0.03$ ]. A risk reduction was also seen for all the components of the primary endpoint (Table 2). The study also showed a reduction in the risk of secondary endpoints (Table 2) including [6]:

- cardiovascular death or hospitalization for HF;

- total number of hospitalizations (initial and recurrent) for HF and cardiovascular deaths;

- all-cause mortality; 
Table 2. Primary and secondary endpoints in the DAPA-HF study (source [6])

\begin{tabular}{|c|c|c|c|c|}
\hline Outcome & $\begin{array}{c}\text { Dapagliflozin } \\
n=2,373\end{array}$ & $\begin{array}{c}\text { Placebo } \\
n=2,371\end{array}$ & $\begin{array}{l}\text { Relative risk } \\
\qquad 95 \% \mathrm{Cl}\end{array}$ & p \\
\hline Primary endpoint & $386(16.3)$ & $502(21.2)$ & $\begin{array}{c}0.74 \\
(0.65-0.85)\end{array}$ & $<0.001$ \\
\hline Hospitalization or urgent visit for HF & $237(10.0)$ & $326(13.7)$ & $\begin{array}{c}0.70 \\
(0.59-0.83)\end{array}$ & - \\
\hline Cardiovascular death & $227(9.6)$ & $273(11.5)$ & $\begin{array}{c}0.82 \\
(0.69-0.98)\end{array}$ & - \\
\hline Hospitalization for HF & $231(9.7)$ & $318(13.4)$ & $\begin{array}{c}0.70 \\
(0.59-0.83)\end{array}$ & - \\
\hline Urgent visit for HF & $10(0.4)$ & $23(1.0)$ & $\begin{array}{c}0.43 \\
(0.20-0.09)\end{array}$ & - \\
\hline \multicolumn{5}{|l|}{ Secondary endpoints } \\
\hline $\begin{array}{l}\text { Cardiovascular death } \\
\text { or hospitalization for HF }\end{array}$ & $382(16.1)$ & 495 (20.9) & $\begin{array}{c}0.75 \\
(0.65-0.85)\end{array}$ & $<0.001$ \\
\hline $\begin{array}{l}\text { All hospitalizations for HF } \\
\text { and cardiovascular deaths }\end{array}$ & 567 & 742 & $\begin{array}{c}0.75 \\
(0.65-0.88)\end{array}$ & $<0.001$ \\
\hline All-cause death & $276(11.6)$ & 329 (13.9) & $\begin{array}{c}0.83 \\
(0.71-0.97)\end{array}$ & $0.022 * *$ \\
\hline Change in $\mathrm{KCCQ}$ * at 8 months & $6.1 \pm 18.6$ & $3.3 \pm 19.2$ & $\begin{array}{c}1.18 \\
(1.11-1.26)\end{array}$ & $<0.001$ \\
\hline Renal function worsening & $28(1.2)$ & $39(1.6)$ & $\begin{array}{c}0.71 \\
(0.44-1.16)\end{array}$ & - \\
\hline
\end{tabular}

*Kansas City Cardiomyopathy Questionnaire (KCCQ) score 0 to 100, with high scores indicating lower severity of heart failure (HF) symptoms; **[7]; $\mathrm{Cl}-$ confidence interval

- improvement in the quality of life as measured using the Kansas City Cardiomyopathy Questionnaire (KCCQ) at 8 months compared to baseline;

- worsening of renal function, defined as persistent eGFR reduction by $\geq 50 \%$, occurrence of end-stage renal failure (defined as permanent eGFR reduction to $<15 \mathrm{~mL}$ / /min/1.73 $\mathrm{m}^{2}$ ), chronic dialysis therapy, or kidney transplantation), or death due to renal causes.

Improved clinical outcomes in the DAPA-HF study were seen in both patients with diabetes type 2 and non-diabetic patients. Dapagliflozin is the only SGLTi that reduced cardiovascular and all-cause mortality in patients with HFrRF (by $18 \%$ and $17 \%$, respectively). Detailed results of the DAPA-HF study are shown in Table 2.

The benefits of dapagliflozin in regard to the primary endpoint rate were similar regardless of the left ventricular ejection fraction, aetiology of HF, concomitant guideline-recommended $\mathrm{HF}$ treatment in terms of drug classes used and medication doses, use of device therapy (ICD/ /CRT) and duration of the disease. The patients with long-standing HF (duration > 5 years) were also shown to benefit from dapagliflozin therapy [8]. The beneficial effects of the drug were seen in all NYHA classes and were greater in milder disease, i.e., in patients in NYHA class II. Thus, dapagliflozin therapy should be initiated early and not postponed until more severe HF symptoms develop.
The benefits of dapagliflozin were similar in all baseline systolic blood pressure categories ( $p$ for interaction 0.78 ) but patients with higher systolic blood pressure, i.e., $\geq 130 \mathrm{~mm} \mathrm{Hg}$, benefited more (HR 0.67, 95\% Cl: 0.60-0.97) compared to patients in the lowest systolic blood pressure category, i.e., with systolic blood pressure < $110 \mathrm{~mm} \mathrm{Hg}$ (HR 0.76, 95\% Cl: 0.51-0.87) [9].

In the DAPA-HF study, absolute risk reduction for the primary endpoint was greatest in the group with a history of a hospitalization for HF within 12 months before inclusion into the study [absolute risk reduction (ARR) 9.9\%], compared to patients hospitalized for HF more than 12 months before inclusion into the study (ARR 4.1\%) and patients without a history of hospitalization for HF (ARR 2.1\%, p for interaction 0.052). These findings mean that the treatment with dapagliflozin should be initiated early after a hospitalization for HF [10].

Based on the DAPA-HF study results, dapagliflozin was approved by the European Medicines Agency (EMA) and the Food and Drug Administration (FDA) in 2020 for the management of symptomatic chronic HFrEF. Dapagliflozin, marketed as Forxiga ${ }^{\mathrm{TM}}$, is the first SGLT2 $i$ approved for the management of HFrEF.

The aim of this publication is to provide clinicians with practical guidance regarding the use of dapagliflozin in HFrEF. 


\section{In whom should dapaglifozin be initiated for the management of heart failure?}

According to the summary of product characteristics (SmPC), dapagliflozin is indicated for the treatment of symptomatic chronic HFrEF in adult patients [11]. Based on data from the large U.S. Get With The Guidelines-Heart Failure (GWTG-HF) registry, $81 \%$ patients with HFrEF would quality for dapagliflozin treatment according to its labelling [12].

The optimal candidate for dapagliflozin treatment is characterized by [6]:

- HFrEF with left ventricular ejection fraction of $\leq 40 \%$;

- NYHA class II-IV symptoms of HF, although the experience with dapagliflozin in NYHA class IV patients is limited due to a relatively low number of such patients included in the DAPA-HF study;

- systolic blood pressure of $\geq 95 \mathrm{~mm} \mathrm{Hg}$;

- ongoing standard treatment for HFrRF, i.e., with a beta-blocker, a renin-angiotensin system inhibitor (ACEI/ARB or ARNI), and a MRA if indicated;

- eGFR of $\geq 30 \mathrm{~mL} / \mathrm{min} / 1.73 \mathrm{~m}^{2}$.

In patients with HFrEF, dapagliflozin dose does not have to be modified if renal dysfunction is present [9]. However, the experience with the use of dapagliflozin in the treatment of HF in patients with severe renal dysfunction (eGFR $<30 \mathrm{~mL} / \mathrm{min} / 1.73 \mathrm{~m}^{2}$ ) is limited.

There is also no need for drug dose adjustment in patients with mild to moderate hepatic dysfunction [11].

\section{How to initiate dapagliflozin treatment?}

Experts recommend that dapagliflozin treatment should be initiated early, optimally before the hospital discharge or during a follow-up visit directly after hospital discharge following hospitalization for exacerbated HFrEF [13]. Dapagliflozin may be added to any chronic HFrEF therapy [6], including drug therapy and device therapy, and regardless of the drug doses used. According to the $\mathrm{SmPC}$, the recommended dapagliflozin dose in HFrEF is $10 \mathrm{mg}$ once daily. The drug may be administered at any time of the day, regardless of meals (either with a meal or between meals).

The drug may be given regardless of the diabetes status and diabetes treatment. However, sulphonylurea and/or insulin dose reduction by about 30\% should be considered in patients receiving concomitant treatment with these drugs due to a risk of hypoglycaemia [9]. Of note, SGLT2i may be insufficiently effective for blood glucose lowering with eGFR below $45 \mathrm{~mL} / \mathrm{min} / 1.73 \mathrm{~m}^{2}$ and other antidiabetic medications should be considered in such patients to control their blood glucose levels [11].

\section{Safety of dapagliflozin treatment}

Dapagliflozin has been used for the treatment of diabetes type 2 for 8 years, and more than 2.5 million patients received this drug in 2019 [14]. Several years of experience, including clinical trials and real-world evidence (RWE), indicate that dapagliflozin is a safe drug. Adverse events $(A E)$, serious $A E$, and $A E$ leading to discontinuation of dapagliflozin treatment are rare, occurring with the rates similar to the rates in the placebo group [6]. The safety data including $A E$ rates are shown in Table 3.

The most common AE categories in the DAPA-HF study were hypovolaemia and renal events (including serious $A E$ : 38 in the dapagliflozin group vs. 65 in the placebo group, $p=0.009$ ) [6], while genital and urinary infections were a marginal problem.

According to the SmPC, there is no need to discontinue dapagliflozin treatment in case of a mild to moderate fungal urogenital infection. Local antifungal therapy or administering a single dose of oral antifungal drug is recommended.

The DAPA-HF study subgroup analyses indicate that dapagliflozin was safe and well tolerated regardless of gender, concomitant diabetes, renal function, and age category [6]. In patients 75 years of age and over, serious renal $\mathrm{AE}$ were less frequent in the dapagliflozin group compared to the placebo group (P for interaction 0.031) [15]. Also in patients with eGFR below $60 \mathrm{~mL} / \mathrm{min} / 1.73 \mathrm{~m}^{2}$ treated with dapagliflozin, the risk of serious $A E$ was significantly lower compared to the placebo group $(p=0.03)$ [16].

In the DAPA-HF study, only rare serious hypoglycaemia and diabetic ketoacidosis events were noted, exclusively in patients with diabetes type 2 (with the rates of $0.4 \%$ and $0.3 \%$, respectively) [6].

Of note in the context of standard HFrRF treatment, dapagliflozin may reduce the risk of moderate to severe hypokalaemia in patients treated with MRA [17].

\section{Practical guidance}

Due to possible hypovolaemia and hypotension during dapagliflozin treatment, the patients should be advised that a modification of diuretic, other antihypertensive drug, and HF drug therapy doses may be required, and adequate fluid intake should be maintained. The risk of symptomatic hypovolaemia and hypotension is increased in those with a history of hypotension, and in the elderly patients [11].

During dapagliflozin treatment, glucosuria is detected on urinalysis, which is consistent with the drug's mechanism of action. Urinary glucose excretion may be associated with an increased risk of external urogenital organ infection, and less frequently urinary tract infection. 
Table 3. Adverse events in the DAPA-HF study [6]

\begin{tabular}{|c|c|c|c|}
\hline Adverse event, n [\%] & $\begin{array}{c}\text { Dapagliflozin } \\
n=2,368\end{array}$ & $\begin{array}{c}\text { Placebo } \\
n=2,368\end{array}$ & p \\
\hline Hypovolaemia & $178(7.5)$ & $162(6.8)$ & 0.40 \\
\hline Renal function-related adverse event & $153(6.5)$ & $170(7.2)$ & 0.36 \\
\hline Fracture & $49(2.1)$ & $50(2.1)$ & 1.00 \\
\hline Amputation & $13(0.5)$ & $12(1.3)$ & 1.00 \\
\hline Severe hypoglycaemia & $4(0.2)$ & $4(0.2)$ & $\mathrm{n} / \mathrm{a}$ \\
\hline Diabetic ketoacidosis & $3(0.1)$ & 0 & $\mathrm{n} / \mathrm{a}$ \\
\hline Fournier gangrene & 0 & $1(<0.1)$ & $\mathrm{n} / \mathrm{a}$ \\
\hline Severe urinary system infection & $14(0.6)$ & $17(0.7)$ & \\
\hline Severe genital organ infection & 0 & $1(0.0)$ & \\
\hline Adverse event leading to drug discontinuation & $111(4.7)$ & $116(4.9)$ & 0.79 \\
\hline
\end{tabular}

Discontinuation of dapagliflozin treatment should be considered in case of pyelonephritis or urosepsis. During dapagliflozin treatment, patients should be advised to pay attention to maintaining adequate urogenital hygiene, including daily underwear changes, wearing non-tight cotton undergarments, and avoiding irritants and scented detergents.

The following changes were noted during dapagliflozin treatment in the DAPA-HF study [6]:

- systolic blood pressure lowering (on average by $1.92 \mathrm{~mm} \mathrm{Hg}$ );

- N-terminal pro-B-type natriuretic peptide (NT-proBNP) level lowering (on average by $196 \mathrm{pg} / \mathrm{mL}$ );

- weight reduction (on average by $0.88 \mathrm{~kg}$ );

- haemoglobin $\mathrm{A}_{1 \mathrm{c}}$ level reduction in diabetic patients (on average by $0.21 \%)$;

- increase in haematocrit (on average by $2.31 \%$ );

- increase in serum creatinine level (on average by $0.07 \mathrm{mg} / \mathrm{dL}$ ).

A transient eGFR reduction may be initially expected during dapagliflozin treatment, particularly in the first 2 weeks. This phenomenon is related to afferent arteriolar constriction due to the tubuloglomerular feedback triggered by an increased sodium influx to the macula densa. During further follow-up in the DAPA-HF study, the rate of eGFR changes in the dapagliflozin group was lower compared to the placebo group, reflecting a nephroprotective effect. Over the entire duration of follow-up, the mean eGFR reduction by about $4 \mathrm{~mL} / \mathrm{min} / 1.73 \mathrm{~m}^{2}$ was documented, while eGFR at 12 months was similar in both study groups [6].

Renal function should be evaluated prior to the initiation of dapagliflozin treatment and monitored thereafter, with serum creatinine level measurement and eGFR calculation at least annually. If eGFR is reduced below $30 \mathrm{~mL} / \mathrm{min} / 1.73 \mathrm{~m}^{2}$ during dapagliflozin treatment, it is recommended to evaluate volume status, blood pressure, and other reversible factors that might contribute to renal function worsening (e.g., other medications, imaging with the use of a contrast agent), correct any contributing factors, and perform follow-up eGFR determination with an individual decision whether to continue dapagliflozin treatment in given clinical settings.

\section{Diabetic ketoacidosis}

Diabetic ketoacidosis during treatment with SGLT2i does not occur in non-diabetic patients with $\mathrm{HFrEF}$, and is a rare occurrence in patients with diabetes type 2 (in the DAPA-HF study, only 3 such cases were identified among patients with diabetes type 2 treated with dapagliflozin) [6], although it may occur with only mildly elevated blood glucose values. It is more frequent during insulin therapy, with poor blood glucose control, and during the first two months of therapy. Diabetic ketoacidosis may be a life-threatening condition. Patients should be educated regarding the symptoms of diabetic ketoacidosis and the need to contact a physician and discontinue SGLT2 $i$ therapy should these symptoms occur. Reinitiation of SGLT2i therapy is possible following stabilization of the clinical condition and reduction of ketonemia (ketone bodies should be measured in blood), when some other obvious cause for diabetic ketoacidosis has been identified and corrected.

Symptoms of diabetic ketoacidosis include nausea, vomiting, anorexia, abdominal pain, excessive thirst, rapid and deep breathing with sweet and fruity breath odour, confusion, and atypical fatigue or somnolescence.

The patients should be evaluated for the risk of diabetic ketoacidosis which is increased in the following situations: - conditions leading to reduced oral food intake or severe dehydration;

- sudden fall in insulin level; 
- increased insulin requirement due to acute illness, surgery, or alcohol abuse;

- low beta cell functional reserve, e.g., in patients with diabetes type 2 and low peptide $C$ level or latent autoimmune diabetes in adults and in patients with a history of pancreatitis.

\section{Precautions}

Dapagliflozin should be used with caution in the following situations:

- severe renal dysfunction;

- history of diabetic ketoacidosis;

- in case of recurrent genitourinary infections;

- the treatment should be interrupted in patients with diabetes type 2 hospitalized due to a major acute illness and before surgical procedures (with drug withdrawal for 3 days before the procedure).

Alcohol abuse and use of ketogenic diets are contraindicated during dapagliflozin treatment.

\section{Contraindications to dapagliflozin}

According to the SmPC [11], use of Forxiga ${ }^{\mathrm{TM}}$ is contraindicated in patients with hypersensitivity to the active substance or any other component of this product. Tablets contain lactose. The drug should not be used in patients with rarely occurring hereditary galactose intolerance, total lactase deficiency or glucose-galactose malabsorption syndrome. Dapagliflozin is also contraindicated in the second and third trimester of pregnancy.

\section{Summary}

Sodium-glucose cotransporter type 2 inhibitors have become a breakthrough in the management of patients with HFrEF. Dapagliflozin is the first SGLT2i approved for the treatment of chronic HFrEF. It is a well-tolerated, safe, and easy-to-use drug. Benefits of dapagliflozin treatment have been documented regardless of standard HFrEF therapies, drug doses, and device therapy, indicating a complementary effect of dapagliflozin over other HFrEF therapies. Dapagliflozin modifies the clinical course of HFrEF, reduces the rate of hospitalizations for HF, cardiovascular deaths, and all-cause deaths, and improves the quality of life of patients with HFrEF regardless of concomitant presence of diabetes type 2.

\section{Conflict of interests}

Lectures for AstraZeneca.

\section{References}

1. Ministerstwo Zdrowia. Mapy potrzeb zdrowotnych. http://mpz.mz.gov. pl/mapy-kardiologiczne/\#tab-2018 (September 15, 2020).

2. Verma S, McMurray JJV. SGLT2 inhibitors and mechanisms of cardiovascular benefit: a state-of-the-art review. Diabetologia. 2018; 61(10): 2108-2117, doi: 10.1007/s00125-018-4670-7, indexed in Pubmed: 30132036.

3. Kaneto $\mathrm{H}$, Obata A, Kimura T, et al. Beneficial effects of sodium-glucose cotransporter 2 inhibitors for preservation of pancreatic $\beta$-cell function and reduction of insulin resistance. J Diabetes. 2017; 9(3): 219-225, doi: 10.1111/1753-0407.12494, indexed in Pubmed: 27754601.

4. Heerspink HJL, Perkins BA, Fitchett DH, et al. Sodium glucose co-transporter 2 inhibitors in the treatment of diabetes mellitus: cardiovascular and kidney effects, potential mechanisms, and clinical applications. Circulation. 2016; 134(10): 752-772, doi: 10.1161/ /CIRCULATIONAHA.116.021887, indexed in Pubmed: 27470878.

5. Heerspink HJL, Kosiborod M, Inzucchi SE, et al. Renoprotective effects of sodium-glucose cotransporter-2 inhibitors. Kidney Int. 2018; 94(1): 26-39, doi: 10.1016/j.kint.2017.12.027, indexed in Pubmed: 29735306.

6. McMurray JJV, Solomon SD, Inzucchi SE, et al. Dapagliflozin in patients with heart failure and reduced ejection fraction. N Engl J Med. 2019; 381(21): 1995-2008, doi: 10.1056/NEJMoa1911303.

7. McMurray JJV, Solomon S, Docherty K, et al. The Dapagliflozin And Prevention of Adverse outcomes in Heart Failure trial (DAPA-HF) in context. Eur Heart J. 2020; 42(13): 1199-1202, doi: 10.1093/eurheartj/ehz916.

8. Yeoh SuE, Dewan P, Jhund PS, et al. DAPA-HF Investigators and Committees. Patient characteristics, clinical outcomes, and effect of dapagliflozin in relation to duration of heart failure: is it ever too late to start a new therapy? Circ Heart Fail. 2020; 13(12): e007879, doi: 10.1161/ /CIRCHEARTFAILURE.120.007879, indexed in Pubmed: 33164553.

9. Serenelli M, Böhm M, Inzucchi SE, et al. Effect of dapagliflozin according to baseline systolic blood pressure in the Dapagliflozin and Prevention of Adverse Outcomes in Heart Failure trial (DAPA-HF). Eur Heart J. 2020; 41(36): 3402-3418, doi: 10.1093/eurheartj/ehaa496, indexed in Pubmed: 32820334.

10. Berg DD, Jhund PS, Docherty KF, et al. Time to clinical benefit of dapagliflozin and significance of prior heart failure hospitalization in patients with heart failure with reduced ejection fraction. JAMA Cardiol. 2021 [Epub ahead of print], doi: 10.1001/jamacardio.2020.7585, indexed in Pubmed: 33595593.

11. Charakterystyka Produktu Leczniczego Forxiga. https://www.astrazeneca.pl/content//dam/az-pl/SPC/SPC_Forxiga_10mg.pdf (March 26, 2021).

12. Vaduganathan M, Greene SJ, Zhang S, et al. Applicability of US Food and Drug Administration labeling for dapagliflozin to patients with heart failure with reduced ejection fraction in US clinical practice: the Get With the Guidelines-Heart Failure (GWTG-HF) Registry. JAMA Cardiol. 2020 [Epub ahead of print], doi: 10.1001/jamacardio.2020.5864, indexed in Pubmed: 33185662.

13. Nessler J, Siniarski A, Leszek P, et al. Expert opinion of the Heart Failure Working Group of the Polish Cardiac Society on the use of dapagliflozin in the treatment of heart failure with reduced ejection fraction. Kardiol Pol. 2021; 79(3): 363-370, doi: 10.33963/KP.15859, indexed in Pubmed: 33687868. 
14. JRaz I. DECLARE-TIMI 58 Trial Rationale, Design, Outcome Selection, Baseline Characteristics, and Effect on Cardiovascular Risk Factors. Presented at ADA 79th Scientific Sessions; June 7-11, 2019; San Francisco.

15. Martinez FA, Serenelli M, Nicolau JC, et al. Efficacy and safety of dapagliflozin in heart failure with reduced ejection fraction according to age: insights from DAPA-HF. Circulation. 2020; 141(2): 100-111, doi: 10.1161/CIRCULATIONAHA.119.044133, indexed in Pubmed: 31736328.
16. Solomon SD, Jhund P, Kosiborod MN et al. Effect of Dapagliflozin on Renal Function in Heart Failure with Reduced Ejection Fraction: The DAPA-HF Trial. Presented at ASN Kidney Week, November 5-10, 2019, Washington.

17. Kristensen SL, Docherty KF, Jhund PS, et al. Dapagliflozin reduces the risk of hyperkalaemia in patients with heart failure and reduced ejection fraction: a secondary analysis DAPA-HF. Eur Heart J. 2020; 41(Suppl_2), doi: 10.1093/ehjci/ehaa946.0939. 